\title{
Special issue on emerging technologies for the management of diabetes mellitus
}

\author{
Konstantia Zarkogianni ${ }^{1} \cdot$ Konstantina S. Nikita $^{2}$
}

Published online: 26 November 2015

(C) International Federation for Medical and Biological Engineering 2015

\section{Introduction}

Diabetes mellitus (DM) is group of chronic metabolic diseases characterized by elevated blood glucose levels resulting from defects in insulin secretion (type $1 \mathrm{DM}-\mathrm{T} 1 \mathrm{DM}$ ), insulin action or a combination of them (type $2 \mathrm{DM}-$ T2DM). DM has serious and mortality-related complications including cardiovascular disease, neuropathy, retinopathy and nephropathy. According to the International Diabetes Federation (IDF), in 2014, 387 million people worldwide suffered from DM, while it is estimated that by 2035 this number will rise to 592 million. A number of 4.9 million deaths were attributed to DM, and the associated annual cost in health expenditure was estimated at USD 612 billion, which corresponded to $11 \%$ of total spending in adults [6].

The rapidly growing number of patients with chronic diseases like DM, along with the escalated costs of treatment and care, the predictable number of deaths and medical errors, poses the urgent need to improve chronic disease management by focusing on preventive, personalized and citizen-centered healthcare systems [16]. Optimal management of DM requires deep understanding of the risk factors associated with the disease, prevention with appropriate

Konstantina S. Nikita

knikita@ece.ntua.gr

Konstantia Zarkogianni

kzarkog@biosim.ntua.gr

1 Biomedical Simulations and Imaging Laboratory, National Technical University of Athens, Athens, Greece

2 Biomedical Simulations and Imaging Laboratory, \& Radio Communications Laboratory, National Technical University of Athens, Athens, Greece lifestyle interventions, early diagnosis and treatment of diabetes before the occurrence of complications, and tight glycemic control $[14,16]$. The ultimate goal is to focus on applying science and technology to enable the creation of new and cost-effective tools to help people with DM. Exploitation of information and communication technologies (ICT) can assist in reshaping the current system of healthcare delivery by shifting the emphasis from the disease to wellness.

\section{Significance of the area}

Recent advancements in wireless sensing and smart devices coupled with smart data analytics have great potential to optimize the management of DM though the following multifold focus: (1) identification of biomarkers, which are strongly related to the onset and the progress of DM, (2) detection of DM at its early stages, (3) patient stratification and (4) tight glycemic control through patients' active participation [16]. Sensor-based technologies for continuous glucose monitoring (CGM) are considered the most significant breakthrough in diabetes management in the past 40 years by providing information regarding patient's glucose profile in response to treatment and lifestyle. Major diabetes-related challenges can be addressed through the integration of different types of data acquired from: (1) wireless pervasive environments facilitating the monitoring of physiological and lifestylerelated parameters, (2) electronic health records (EHR), which include demographic, clinical, treatment and medical history information, (3) patient's genetic profile and (4) patient's biological state, which can be inferred by combining genomic, transcriptomic, epigenomic, proteomic and metabolomics data. 
As more and more data are gathered, data processing and interpretation become more crucial in order to transform the acquired raw data into clinically meaningful knowledge with the ultimate goal to support diabetes decision making. The application of advanced modeling, control, classification and clustering methodologies on different combinations of datasets has led to the development of a range of clinical decision support systems (CDSS) [16]. Reliable computational models able to predict glucose profile in response to various stimuli and insulin infusion advisory algorithms able to produce personalized recommendations of insulin rates/injections are assuming to provide valuable tools within the context of achieving tight glycemic control in patients with DM [15]. Computer-based risk prediction models for the incidence of long-term diabetes complications have been proposed, and their potential to support clinical decision making toward initiating appropriate treatment has been demonstrated [12]. Models able to detect T2DM at its early stages and identify people at an increased risk of developing the disease are also of paramount importance [13].

The aforementioned CDSS constitute the key modules toward the development of integrated systems and services facilitating self-disease management and supporting healthcare professionals in decision making. Multi-parametric monitoring systems combined with intelligent interoperable communication platforms enable remote monitoring, context awareness, integrative risk assessment, as well as automated closed-loop insulin delivery $[11,16]$. Within the same context, artificial pancreas (AP) is one of the major challenges for the management of insulin-treated patients with DM. It is a closed-loop blood glucose control system that combines a glucose sensor, a control algorithm and an insulin infusion pump [4, 14].

\section{Overview of the special issue}

This special issue includes nine research articles presenting highly innovative activities which reveal the rapidly changing face and context of diabetes monitoring and management facilitated by advances in sensing and drug delivery technologies, simulation and control algorithms, and health information technologies. Novel solutions for monitoring essential physiological parameters along with current trends in drug delivery systems and CDSS within the context of diabetes management constitute the main focus of the special issue.

The paper authored by Cobelli et al. [1] proposes a CGM sensor error model focusing on two commercially available sensors both belonging to the recently presented "fourth" generation of Dexcom CGM sensors. CGM readings are still subject to inaccuracies caused by the unavoidable random measurement noise, distortions due to the blood-to-interstitial glucose kinetics and under-/overestimations associated with the sensor calibration process. Having as ultimate goal to quantify and simulate those error components, the analysis presented by the authors reveals important features such as the CGM sensor error's time variance during the monitoring and can provide the basis toward the development of new reliable CGM sensor error models to be incorporated into glucose predictors and virtual environments for testing closed-loop glucose controllers.

The paper by Fotiadis et al. [3] addresses the challenge to identify the most informative features, as extracted from medical and lifestyle self-monitoring data with respect to their ability to predict the short-term subcutaneous (s.c.) glucose concentration. Toward this direction, a combination of feature ranking and regression models is applied. Glucose profile, time of the day and plasma insulin concentration constitute important features, while the effect of food intake and physical activity is different among patients.

Zarkogianni et al. [17] investigate the ability to produce accurate glucose profile predictions based on data provided by only two sensors, monitoring glucose concentration and physical activity, respectively. Issues related to low-input dimensionality, low complexity, stability and subsequent integration of the glucose prediction models in closedloop glucose controllers have motivated the application of three different data-driven modeling techniques based on feedforward neural network (FNN), self-organizing maps (SOM) and neuro-fuzzy network with wavelets as activation functions (WFNN). The presented results demonstrate SOM's superiority and justify the need to apply sophisticated modeling techniques toward the development of reliable personalized glucose prediction models.

Telemedicine and mobile health systems can play an important role in the management of DM providing costeffective solutions for remote monitoring and patient empowerment. The paper by Lanzola et al. [7] describes two platforms that are developed and tested within the framework of the AP@home project in order to support the exploitation of an artificial pancreas system. The PC-based platform enables the evaluation of the insulin infusion advisory algorithms, while the mobile-based platform aims at improving the comfort for the patient. Martinez-Millana et al. [8] propose an integrated system for the remote monitoring of patients with DM based on physiological sensors, mobile technologies and patient/doctor applications over a single service-oriented architecture. The system is tested under real-life conditions and evaluated in terms of data sustainability and runtime performance of clients. Fioravanti et al. [2] present a new strategy for $m$-health applications in diabetes self-management, based on automatic generation of feedback messages. Preliminary results obtained by conducting a small-scale exploratory study show increased user acceptance and adherence to prescription. 
Prolonged diabetes retinopathy leads to diabetic macular edema (DME) which is the most common cause of visual loss among patients with DM. Early detection of DME is of paramount importance in order to initiate appropriate treatment and improve visual acuity. The paper co-authored by Mookiah [9] is focused on automated grading of DME based on higher-order spectra (HOS) of Radon transform projections of the fundus images. The proposed method demonstrates increased discrimination abilities. The paper by Ibrahim et al. [5] presents the development and evaluation of a data-adaptive neuro-fuzzy inference classifier with the ability to accurately classify diabetes maculopathy images.

Models for the simulation of glucose metabolism can give new insights regarding the underlying pathological mechanisms involved in DM. Toward this direction, compartmental modeling approaches have been applied in order to evaluate the effect of insulin, glucagon and C-peptide on glucose utilization [10]. Villiam Mojito et al. [5] propose a methodological framework based on pharmacokinetic/ pharmacodynamic (PK/PD) modeling strategy, in order to model the effect of C-peptide on glucose utilization after injection of a glucagon bolus in patients with T1DM. The most important benefit of the proposed method relies on the utility of a four-point glucagon stimulation test.

\section{Future trends}

This special issue presents breakthrough research ICTbased activities for the management of DM and elucidates the multi-disciplinary nature of this emerging and important domain. Several open issues and technical challenges offered by modern health information technologies, including electronic health records, web-based patient portals, telemedicine, clinical decision support systems and patient registries, play a crucial role toward the development of strategies to improve diabetes health outcomes. Contributions justify the great progress that has been made in the recent years toward the development of CGM sensor error models, glucose prediction models, artificial pancreas, mobile healthcare systems, early detection of complications and glucose metabolism physiological models. However, several open issues and technical challenges are identified as key factors for fully adopting these systems in clinical practice. Future research directions are summarized in the following:

1. Systematic and reliable evaluation framework The criteria for selecting patients and controls and the approaches for the treatment of controls vary greatly among published evaluation studies leading to biased data analysis which in turn results in producing unre- liable outcomes. On top of this, substantial economic analysis for proving the cost effectiveness of the proposed systems is missing, making thus questionable their applicability. Hence, it is particularly important and challenging to follow a more reliable and in-depth evaluation framework in order to prove the effectiveness of the proposed solutions in terms of user acceptance, technical validation, accuracy, safety and cost.

2. Data security and privacy Data security and privacy is an issue of growing importance. ICT-based solutions must ensure protection of private records containing patient's health status, personal history and medical treatments from disclosure to any unauthorized individual.

3. Unobtrusive sensing Transient critical abnormalities should be early detected since DM pathophysiology is a continuing process. Taking also into account the multifactorial nature of the disease, a multitude of different data types should be monitored. Sensor networks with the ability to monitor physiological (e.g., glucose, blood pressure, pulse and cardiac rhythm) and lifestyle (e.g., diet and physical activity) data can provide essential information to detect such transitions and track the progress of the disease. The emerging technology of internet of things has great potential to contribute in this direction, through permitting global connectivity among sensors and devices, enabling thus seamless integration of more diabetes-related factors.

4. User-centered approaches Holistic user-centered approaches incorporating personalized predictive models coupled with heterogeneous sources of data (patient, clinical, biological, therapeutic, behavioral, physical training and performance, lifestyle and diet, environmental data and social data) can raise individual awareness, promote behavioral lifestyle changes, support treatment and track the progress of the disease. Emphasis should be given in establishing interactions between patients and health professionals within the context of co-decision making and in creating multi-stakeholder ecosystems.

5. Multi-level and multi-scale modeling New powerful multi-level and multi-scale modeling strategies are needed in order to handle and successfully process a multitude of different types of data from behavioral down to molecular level.

\section{References}

1. Facchinetti A et al (2015) Model of glucose sensor error components: identification and assessment for new Dexcom G4 generation devices. Med Biol Eng Comput 53. doi:10.1007/ s11517-014-1226-y

2. Fioravanti A et al (2015) Automatic messaging for improving patients engagement in diabetes management: an exploratory study. Med Biol Eng Comput 53. doi:10.1007/s11517-014-1237-8 
3. Georga E et al (2015) Evaluation of short-term predictors of glucose concentration in type 1 diabetes combining feature ranking with regression models. Med Biol Eng Comput 53. doi:10.1007/ s11517-015-1263-1

4. Heinemann L, Benesch C, DeVries J (2011) AP@ home: a novel European approach to bring the artificial pancreas home. J Diabetes Sci Technol 5(6):1363-1372

5. Ibrahim $S$ et al (2015) Classification of diabetes maculopathy images using data-adaptive neuro-fuzzy inference classifier. Med Biol Eng Comput 53. doi:10.1007/s11517-015-1329-0

6. International Diabetes Federation (2000) IDF diabetes Atlas, 6th edn. http://www.idf.org/diabetesatlas/download-book (Online)

7. Lanzola $G$ et al (2015) Designing an artificial pancreas architecture: the AP@home experience. Med Biol Eng Comput 53. doi:10.1007/s11517-014-1231-1

8. Martinez-Millana A et al (2015) Performance assessment of a closed-loop system for diabetes management. Med Biol Eng Comput 53. doi:10.1007/s11517-015-1245-3

9. Mookiah M et al (2015) Application of higher-order spectra for automated grading of diabetic maculopathy. Med Biol Eng Comput 53. doi:10.1007/s11517-015-1278-7

10. Mojto V et al (2015) Short-term glucagon stimulation test of C-peptide effect on glucose utilization in patients with Type 1 diabetes mellitus. Med Biol Eng Comput 53. doi:10.1007/ s11517-015-1416-2

11. Mougiakakou SG et al (2010) SMARTDIAB: a communication and information technology approach for the intelligent monitoring, management and follow-up of Type 1 diabetes patients. IEEE Trans Inf Technol Biomed 14(3):622-633

12. Stevens RJ et al (2001) The UKPDS risk engine: a model for the risk of coronary heart disease in type 2 diabetes. Clin Sci 101(6):671-679

13. Wilson $\mathrm{P}$ et al (2007) Prediction of incident diabetes mellitus in middle-aged adults: the Framingham Offspring Study. Arch Intern Med 167:1068-1074

14. Zarkogianni K, Nikita KS (2015) Personal health systems for diabetes management, early diagnosis and prevention. In: Fotiadis DI (ed) Handbook of research on trends in the diagnosis and treatment of chronic conditions. Hershey: Medical Information Science Reference, pp. 468-492

15. Zarkogianni $\mathrm{K}$ et al (2011) An insulin infusion advisory system based on autotuning nonlinear model-predictive control. IEEE Trans Biomed Eng 58(9):2467-2477

16. Zarkogianni $\mathrm{K}$ et al (2015) A review of emerging technologies for the management of diabetes mellitus. IEEE Trans Biomed Eng 62(12):2735-2749

17. Zarkogianni $\mathrm{K}$ et al (2015) Comparative assessment of glucose prediction models for patients with type 1 diabetes mellitus applying sensors for glucose and physical activity monitoring. Med Biol Eng Comput 53. doi:10.1007/s11517-015-1320-9

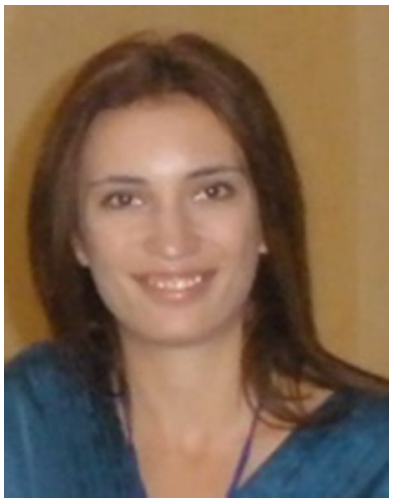

Konstantia Zarkogianni received the diploma in Electrical and Computer Engineering (2003) from the Aristotle University of Thessaloniki, Greece, the M.Sc. degree in Electronic and Computer Engineering (2005) from the Technical University of Crete (TUC), Greece, and the Ph.D. degree (2011) from the National Technical University of Athens (NTUA), Greece. Since 2011, she is doing postdoctoral research at the Biomedical Simulations and Imaging Laboratory (BIOSIM) of
NTUA. She is the author or the co-author of 8 papers published in refereed international journals, 1 chapter in book and 18 papers in international conference proceedings. Her current research interests include medical decision support systems, control systems, simulation of physiological systems and diabetes management. She has served as guest editor for the special issue in emerging technologies for the management of diabetes published in Medical and Biological Engineering and Computing Journal. She is also a reviewer of several international journals. She has participated as a research associate in national and EU-funded projects. She is a member of the Institute of Electrical and Electronics Engineers and the Technical Chamber of Greece.

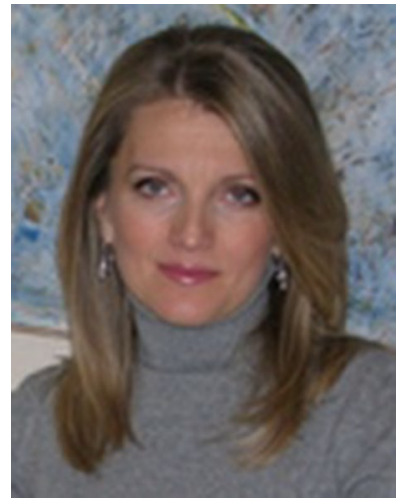

Konstantina S. Nikita received the diploma in Electrical Engineering and the Ph.D. degree from the National Technical University of Athens (NTUA), as well as the M.D. degree from the Medical School, University of Athens. From 1990 to 1996, she worked as a researcher at the Institute of Communication and Computer Systems. In 1996, she joined the School of Electrical and Computer Engineering, NTUA, as an assistant professor, and since 2005, she serves as a professor at the same school. She has authored or co-authored 157 papers in refereed international journals, 41 chapters in books and over 300 papers in international conference proceedings. She is editor of seven books in English and author of two books in Greek. She holds two patents. She has been the technical manager of several European and National R\&D projects. She has been honorary chair/chair of the program/organizing committee of several international conferences, and she has served as keynote/invited speaker at international conferences, symposia and workshops organized by NATO, WHO, ICNIRP, IEEE, URSI, etc. She has been the advisor of 25 completed Ph.D. theses, several of which have received various awards. Her current research interests include biomedical signal and image processing and analysis, simulation of physiological systems, biomedical telemetry, biological effects and medical applications of radiofrequency electromagnetic fields and biomedical informatics. She is a member of the Editorial Board of the IEEE Transactions on Biomedical Engineering, the IEEE Journal of Biomedical and Health Informatics, the Wiley Bioelectromagnetics and the Journal of Medical and Biological Engineering and Computing and a guest editor of several international journals. She has received various honors/awards, with the Bodossakis Foundation Academic Prize (2003) for exceptional achievements in "Theory and Applications of Information Technology in Medicine" being one of them. She has been a member of the Board of Directors of the Atomic Energy Commission and of the Hellenic National Academic Recognition and Information Center, as well as a member of the Hellenic National Council of Research and Technology. She has also served as the Deputy Head of the School of Electrical and Computer Engineering of the NTUA. She is an Adjunct Professor of Biomedical Engineering and Medicine, Keck School of Medicine and Viterbi School of Engineering, University of Southern California. She is a member of the Hellenic National Ethics Committee, a Founding Fellow of the European Association of Medical and Biological Engineering and Science (EAMBES) and a member of the Technical Chamber of Greece and of the Athens Medical Association. She is also the founding chair and ambassador of the IEEE-EMBS, Greece chapter and vice chair of the IEEE Greece Section. 